\title{
CRACK INITIATION AND PROPAGATION DURING THERMAL-MECHANICAL FATIGUE OF IN792: EFFECTS OF DWELL TIME
}

\author{
Paraskevas Kontis $^{1}$, David M. Collins ${ }^{1}$, Sten Johansson ${ }^{2}$, Angus J. Wilkinson ${ }^{1}$, \\ Johan J. Moverare ${ }^{2}$, Roger C. Reed ${ }^{1}$ \\ ${ }^{1}$ Department of Materials, University of Oxford, Parks Road, OX1 3PH Oxford, UK \\ ${ }^{2}$ Engineering Materials, Dept. of Management and Engineering, Linköping University, 58183 Linköping, \\ Sweden
}

Keywords: deformation, carbides, strain mapping

\begin{abstract}
Thermal-mechanical fatigue (TMF) fatigue crack growth measurements are performed on the polycrystalline alloy IN792, in mechanical strain control and in the temperature range $100-750{ }^{\circ} \mathrm{C}$. The effect of both in-phase (IP) and out-of-phase (OP) TMF cycles on the crack growth rate are investigated and the impact of prolonged dwell times at the highest investigated temperature is emphasised. Scanning electron microscopy (SEM) including electron backscatter diffraction (EBSD) techniques are used to characterise the cracking phenomena; cross-correlationbased analysis of EBSD patterns is used to obtain high resolution maps of lattice rotation and elastic strains near second phases such as carbides. The six hours dwell time at maximum temperature increases crack growth rate for both types of TMF; higher rates are observed in the case of IP. MC-type carbides are found to play an important role in crack initiation performance of IN792 by decreasing the crack initiation life, particularly in the IP-tests. Finally, a transition from transgranular to intergranular crack propagation was observed for the IP-test as dwell time increased, the transgranular character of the cracking in the OP-tests was not affected by the dwell time.
\end{abstract}

\section{Introduction}

Resistance to thermal-mechanical fatigue is becoming of increasing importance to hot components for gas turbines, most notably when large temperature gradients exist from engine start-up or shut-down. These yield complex stress and temperature cycles in turbine blades and vanes, and hence TMF damage may be prevalent. The advanced cooling systems used in steady-state operation also produce temperature gradients that may also result in TMF damage [1].

It is well known that a number of different methods are available for monitoring crack growth rates when the temperature is isothermal. However, very few studies report on crack propagation behaviour during thermal cycling and in particular when a dwell period is used. This situation has provided the motivation for the present study. Here, new insights into the effects occurring under such conditions are sought. There is significant industrial relevance: the service life of many hot components is not entirely controlled by the number of cycles to crack initiation - a certain amount of crack propagation may occur prior to component replacement. In this case, prediction schemes for crack growth rates need to be accurate [2].

\section{Experimental Procedures}

The chemical composition of the alloy IN792 investigated is Ni12.4Cr-8.9Co-1.8Mo-4.0W-3.5Al-4.0Ti-4.1Ta-0.08C-0.017B$0.019 \mathrm{Zr}$ (wt.\%). The test material was delivered by Howmet as conventionally cast round bars of diameter $20 \mathrm{~mm}$. The material was hot isostatically pressed (HIP) at $1195^{\circ} \mathrm{C}$ and $150 \mathrm{MPa}$ for $2 \mathrm{~h}$ followed by solution heat treatment at $1121{ }^{\circ} \mathrm{C}$ for $2 \mathrm{~h}$ and then ageing at $850^{\circ} \mathrm{C}$ for $24 \mathrm{~h}$.

Both in-phase (IP) and out-of-phase (OP) thermal-mechanical fatigue tests were conducted under mechanical strain control in the $100-750{ }^{\circ} \mathrm{C}$ temperature range using an MTS 810 servohydraulic thermal-mechanical fatigue machine; the experimental of which has also been described [3].

Single edge notched (SEN) type specimens were machined of the geometry illustrated in Figure 1. To study the crack initiation sites and the crack propagation character during IP and OP-TMF, the specimens were sectioned parallel to the tensile axis as shown in Figure 1. Polished surfaces were prepared metallographically and observed by backscattered electron imaging and electron backscattered diffraction (EBSD). In addition, energy dispersive $\mathrm{X}$-ray (EDX) chemical mapping was conducted using a Zeiss Merlin FEG-SEM.

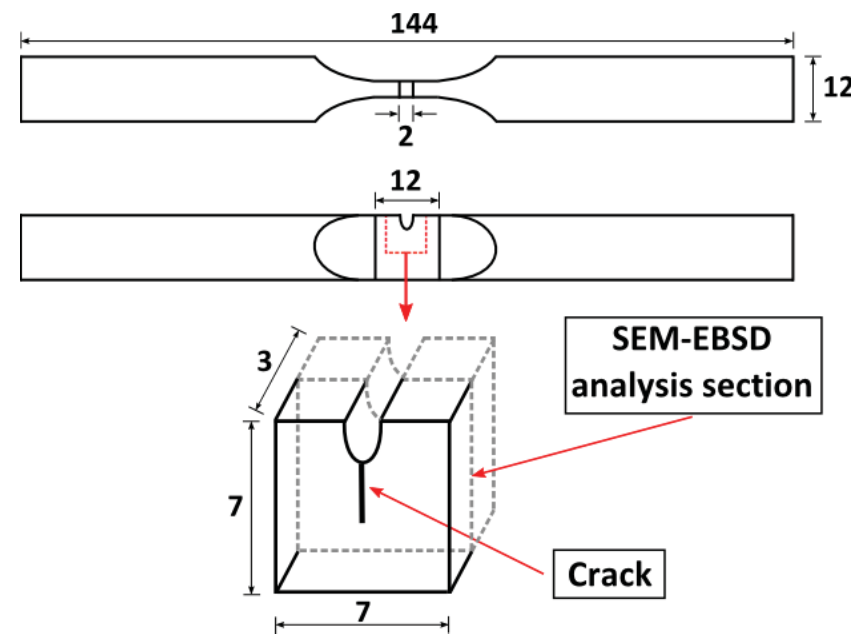

Figure 1: Geometry of single edge notched (SEN) type specimen (dimensions in $\mathrm{mm}$ ). 
An extensometer with a $12 \mathrm{~mm}$ gauge length was attached symmetrically to the specimen over the notch. All tests were conducted in displacement control by prescribing the displacement of the extensometer gauge length. Consistent with common practice, in this work the displacement measured by the extensometer $(\Delta \mathrm{L})$ divided by the extensometer gauge length $\left(\mathrm{L}_{0}\right)$ will be referred to as the nominal total strain $\left(\varepsilon_{\text {nom }}=\Delta \mathrm{L} / \mathrm{L}_{0}\right)$. To enable thermal cycling, induction heating was used in combination with convection cooling from compressed air distributed onto the specimen by two cooling nozzles. To examine the effect of dwell time on TMF crack propagation, hold times of 5 and 360 minutes (6 hours) were applied at the maximum temperature. The test matrix is summarized in Table I. Crack lengths were determined using the compliance method in a similar manner following the standard ASTM E 647 for isothermal crack growth tests [2].

Table I. IP and OP-TMF tests conducted in this study for the investigation of the effect of dwell-time during TMF.

\begin{tabular}{|c|c|c|}
\hline Cycle type & Dwell, min & $\varepsilon_{\text {nom, } \%}$ \\
\hline IP $(\mathrm{R}=0)$ & 5 & 0.6 \\
\hline $\mathrm{OP}(\mathrm{R}=-\infty)$ & 5 & 0.6 \\
\hline $\mathrm{IP}(\mathrm{R}=0)$ & 360 & 0.6 \\
\hline $\mathrm{OP}(\mathrm{R}=-\infty)$ & 360 & 0.6 \\
\hline
\end{tabular}

Finally, to investigate the effect of MC carbides on the TMF life, high resolution EBSD strain mapping was performed for both the non-deformed and deformed specimens. In each case, EBSD patterns were recorded using a $1000 \times 1000$ pixel, peltier cooled charge coupled device (CCD) camera at full resolution on a JEOL-6500 FEG-SEM. Diffraction patterns were collected with a beam of $20 \mathrm{keV}$ energy, a probe current of $15 \mathrm{nA}$, and an acquisition time of $0.5 \mathrm{~s}$. Lattice rotations and elastic strains within the sample cause small shifts in the positions of the zone axes and other features in the EBSD patterns obtained as the electron beam is scanned over the sample. These small pattern shifts are measured using an automated image-processing method based on cross-correlation analysis and then related to the size and nature of the lattice strains and rotations, following [4].

\section{Results}

\section{$\underline{\text { Crack Growth Rates }}$}

Data for crack length as a function of the number of cycles is summarised in Figure 2. Crack initiation occurred rather early in all tests and in all cases a significant number of cycles were consumed in crack propagation. A general observation was that the crack propagation rate decreased with increasing crack length, particularly after the length of the crack reached $1.5 \mathrm{~mm}$. Note that for the OP test with a 5 minutes dwell, some data points are absent due to missed data logging during the test.

The crack growth rate is next calculated from the derivative of the crack length versus number of cycles $(\mathrm{d} a / \mathrm{d} N)$ data; the results of this procedure are plotted in Figure 3. It can be seen that the crack growth rate $(\mathrm{d} a / \mathrm{d} N)$ is slightly higher for the OP condition compared to IP, when the dwell time is $5 \mathrm{~min}$. However, a dwell time of 6 hours at the highest temperature increases the crack growth rate dramatically in both IP and OP conditions; slightly higher crack growth rate was observed in the IP case with dwell.

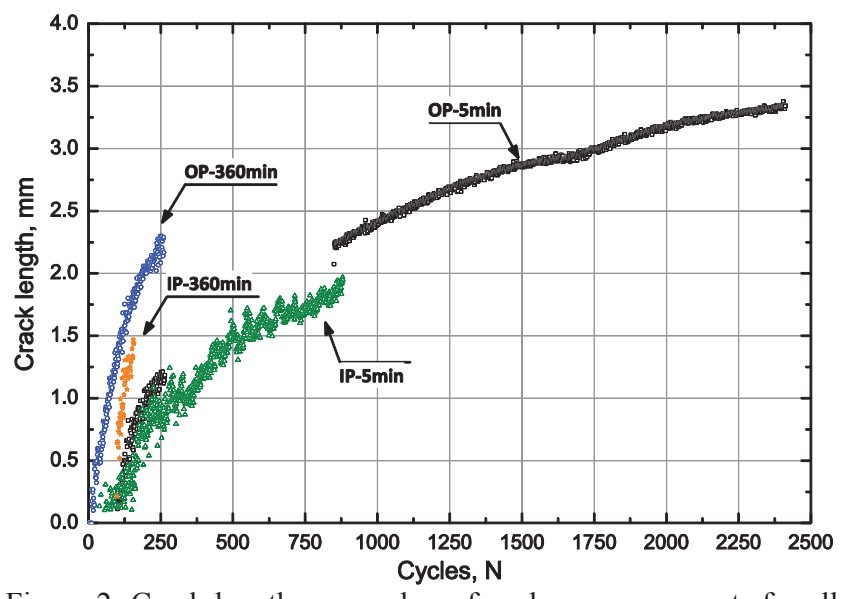

Figure 2: Crack length vs. number of cycles measurements for all the investigated TMF conditions for the IN792 alloy.

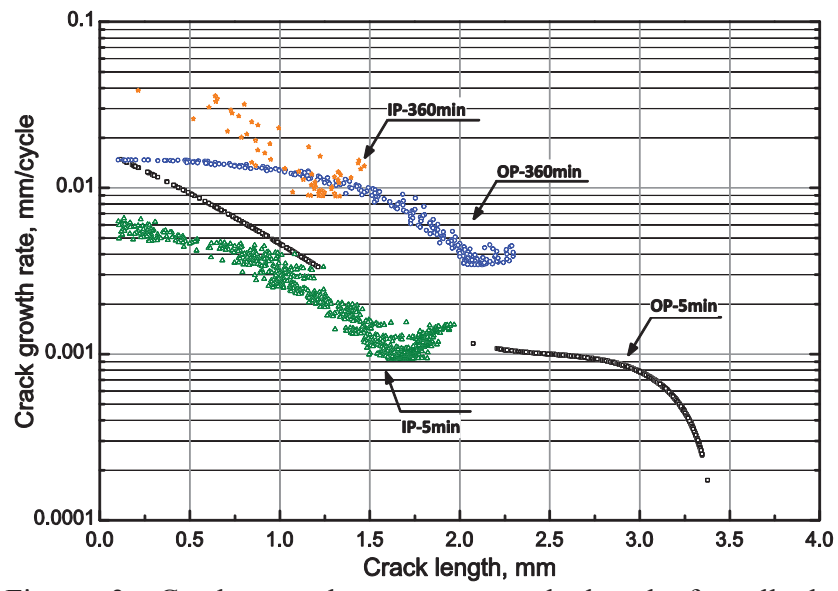

Figure 3: Crack growth rate $v s$. crack length for all the investigated TMF conditions.

The above observation can also be observed in Figure 4, where the crack growth rate is plotted as a function of maximum stress intensity factor. Assuming linear fracture mechanics is valid at elevated temperatures, the stress intensity factor $\mathrm{K}_{\mathrm{I}}$ is defined as:

$$
\mathrm{K}_{\mathrm{I}}=\mathrm{Y} \sigma \sqrt{ }(\pi \alpha)
$$

where $Y$ is the geometry factor and $\alpha$ is the crack length. A relationship between the stress intensity factor, the crack length and the applied force for the particular specimen geometry used in the current study has been established previously [2]. From Figure 4 it is apparent that a 6 hours dwell has dramatically affected the crack growth rate in the IP-tests, whereas the crack growth rate in the OP case remains evident but its influence is weaker. When the results from IP and OP with 6 hours dwell are compared, a relatively higher crack growth rate is observed for the IP-test. 


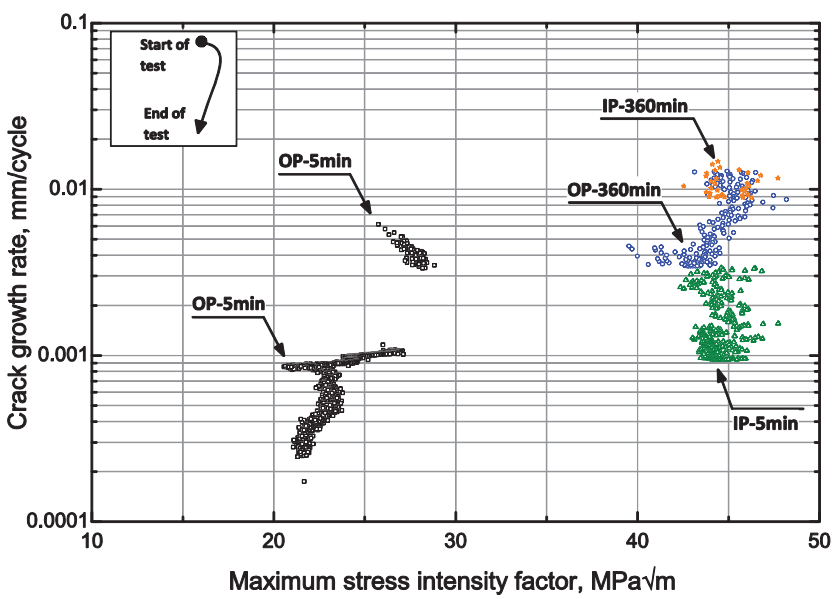

Figure 4: Crack growth rate as a function of the maximum stress intensity factor for all the investigated TMF conditions.

Figure 5 illustrates the minimum and maximum forces as a function of crack length, respectively. Since the tests were performed in displacement control, the force response will change as the length of the crack increases, as observed. In addition, it can be seen that in the case of the 5 minute dwell tests, the maximum force in the IP-case is relatively high compared to the OP-test. However, for the 6 hour dwell test, the maximum force in the OP-test increases substantially; similar behaviour is not observed for the IP-test.

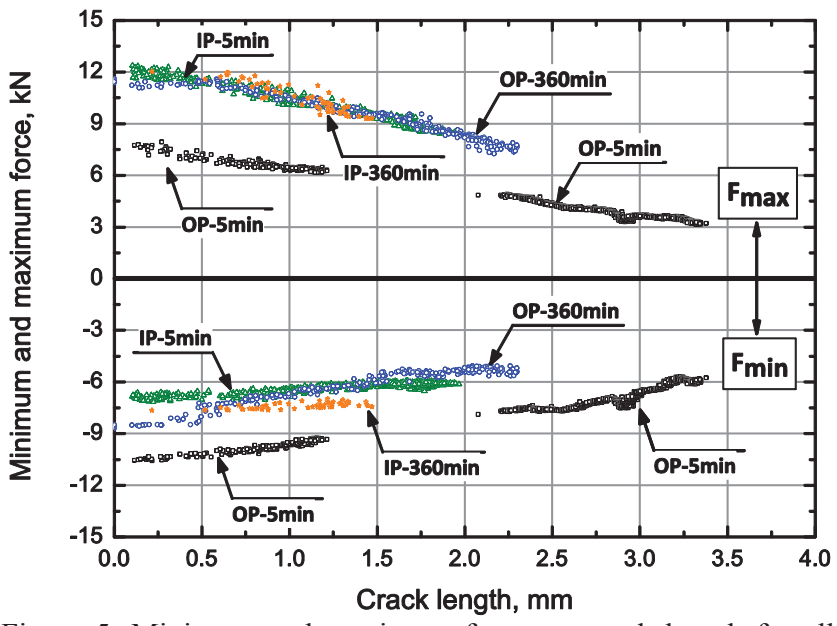

Figure 5: Minimum and maximum force $v s$. crack length for all the investigated TMF conditions.

\section{$\underline{\text { Crack Initiation }}$}

In IN792, it would appear that the TMF crack initiation life is controlled by the MC-type carbides which have precipitated interand transgranularly; MC carbides were found to have cracked or voids have formed at the interfaces between MC and matrix $\left(\gamma / \gamma^{\prime}\right)$ microstructure. Cracks growing due to oxidation damage from the surface and through the $\gamma^{\prime}$ denuded zone caused by it, also need to be taken into account. Table II summarises the parameters found to influence the crack initiation life of IN792 at all the conditions investigated.
Table II. Summary of crack initiation sites for all the investigated conditions.

\begin{tabular}{|c|c|c|}
\hline 5 min dwell & IP-TMF & OP-TMF \\
\hline 360 min dwell & $\begin{array}{c}\text { MC carbides } \\
\text { cracking } \\
\text { goids forming at } \\
\text { goundaries/MC } \\
\text { carbides cracking }\end{array}$ & $\begin{array}{c}\text { Oxidised MC } \\
\text { carbides close to } \\
\text { surface }\end{array}$ \\
\hline Oarbides close to \\
surface
\end{tabular}

In the case of the IP-tests, the MC carbides are considered to have a rather detrimental effect on the crack initiation life. In particular, for the IP-test with a 5 minutes dwell the majority of the MC carbides were observed to have cracked. In addition, voids formed at the MC and $\gamma / \gamma^{\prime}$ interfaces, as illustrated in Figure 6a.

As dwell time increased under IP conditions, the cracks were found to initiate primarily at grain boundaries. However, the MC carbides still exhibit similar behaviour as previously. It is believed that the observed brittle nature of $\mathrm{MC}$ carbides combined with longer dwell times are responsible for the transition to intergranular crack initiation. Figure $6 \mathrm{c}$ illustrates voids forming at grain boundaries and at the grain interior, particularly at the interfaces between $\mathrm{MC}$ and matrix $\left(\gamma / \gamma^{\prime}\right)$ microstructure.

Although the crack initiation sites can be related to oxides forming from the surface, the fact that the majority of $\mathrm{MC}$ carbides are cracked at the IP-tests is the prevailing observation the formation of voids at these particular interfaces in the IP-tests dramatically decreases the crack initiation time of the alloy. MC carbides have been reported to serve as crack initiation sites in previous studies on polycrystalline superalloys particularly during isothermal fatigue studies $[5,6,7,8,9]$.

In order to rationalise the void formation at the $\mathrm{MC} /$ matrix interfaces during IP-testing, strain mapping analysis was conducted for both IP-tests and close to MC carbides. Figure 7 illustrates the investigated MC carbide close to the crack tip alongside corresponding strain maps in the case of IP-test with 6 hours dwell. The accumulation of plasticity around the MC carbide is apparent from the backscattered image (dark contrast regions) and it is also confirmed by the geometrically necessary dislocation (GND) density measurements, as illustrated in Figure 7. This is expected due to the presence of high strain gradients at the interface of MC and $\gamma / \gamma^{\prime}$. If accumulation of plasticity around MC carbides occurs relatively soon compared to oxidation damage in the IP-tests, then the MC carbides will serve as the predominant sites for crack initiation, and we believe this to be the case here. Note that due to the unknown elastic constant of the MC carbide, these regions have not been included in the crosscorrelation analysis routine. These regions, in addition to any point of low diffraction pattern quality have been omitted in Figures 7,8 and 11. 

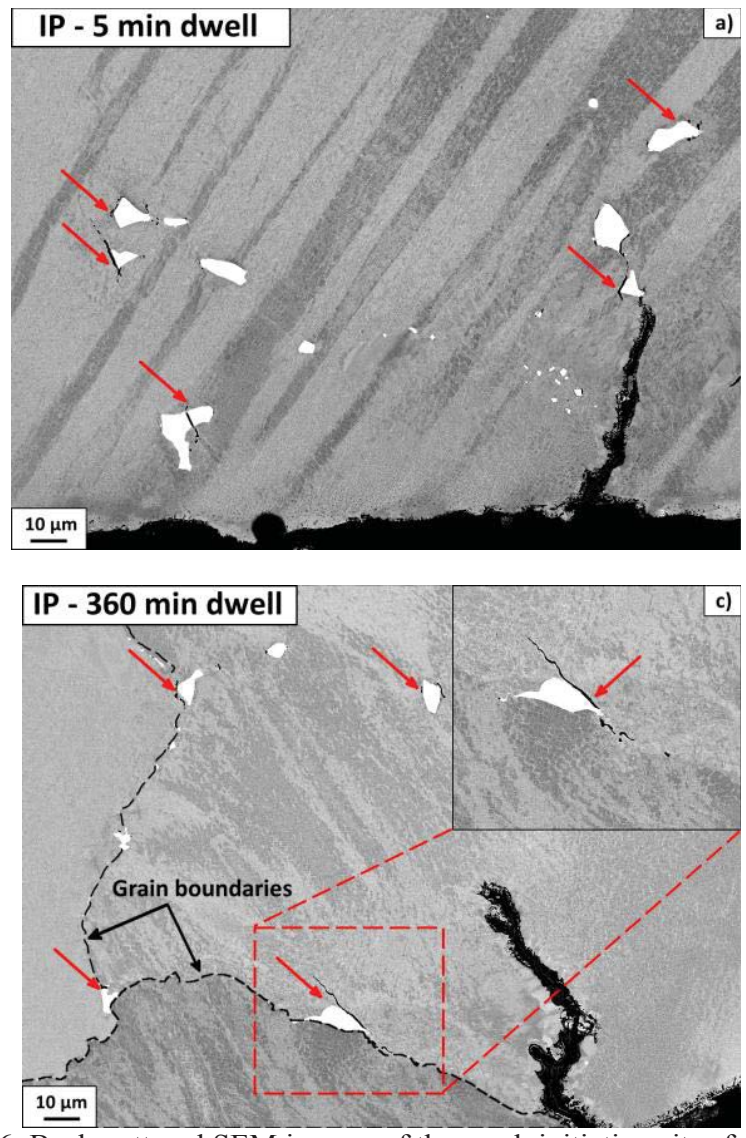

Figure 6: Backscattered SEM images of the crack initiation sites for a) IP-5 minutes dwell, b) OP-5 minutes dwell, c) IP-360 minutes dwell and d) OP-360 minutes dwell specimens. Load axis is in horizontal direction. The box illustrates the area scanned for EDX mapping see Figure 8). Red arrows indicate voids around or cracks within MC carbides.
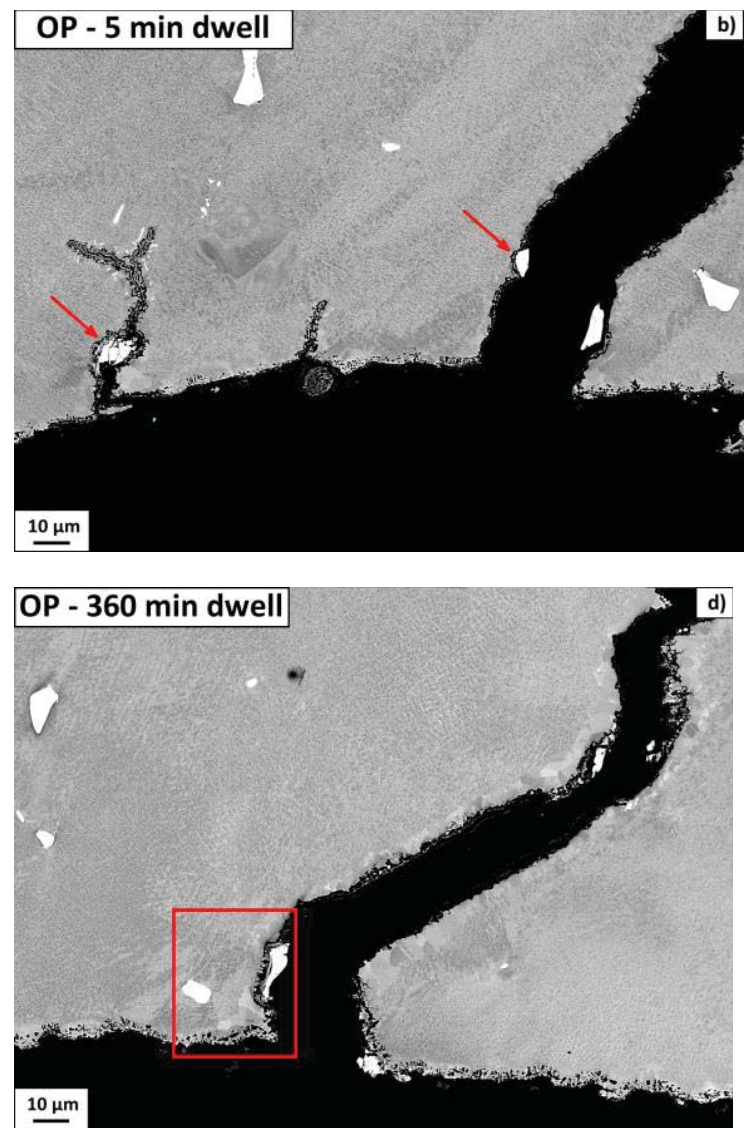

\section{d)}


Moreover, Figure 8 illustrates a backscattered SEM micrograph from a carbide from the IP-test with 5 minutes dwell. Large lattice rotations can be observed in the vicinity of voids, corresponding to regions of high dislocation density shown in the GND map. It is apparent that $\mathrm{MC}$ carbides cannot accommodate plastic deformation and thus voids initiate at the interface of $\mathrm{MC}$ with the surrounding matrix $\left(\gamma / \gamma^{\prime}\right)$ microstructure.
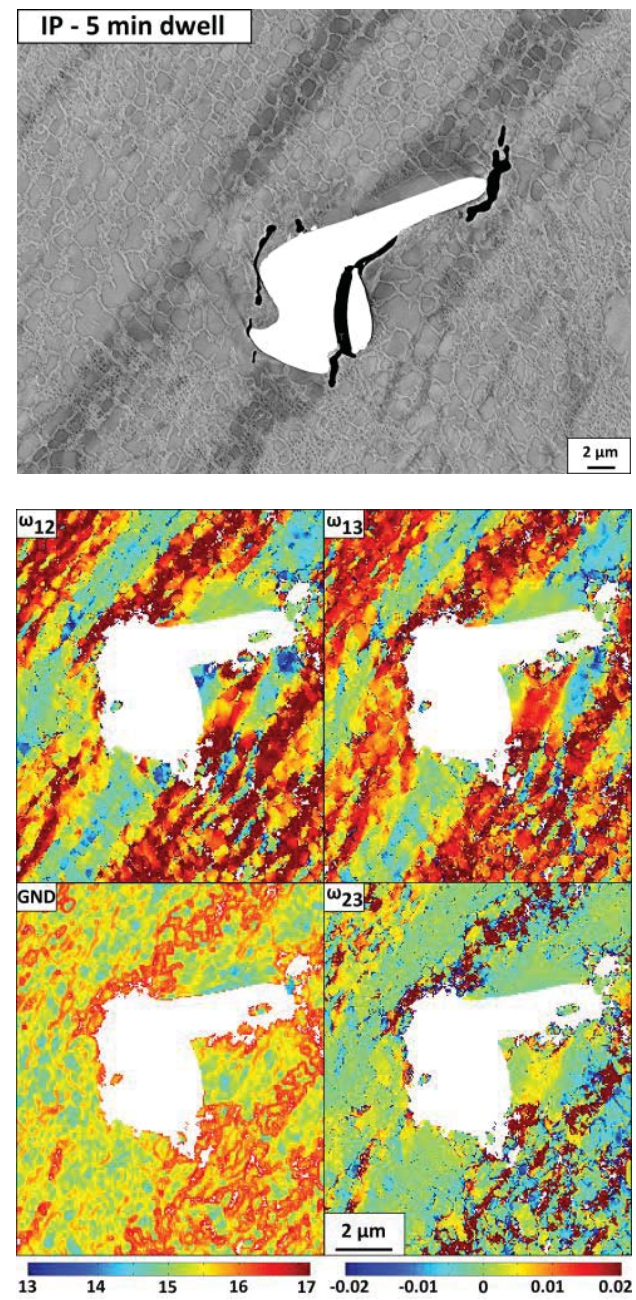

Figure 8: Backscattered SEM image of cracked MC carbide from the IP-test with 5 minutes dwell, with corresponding lattice rotations maps (rotations in radians) and geometrically necessary dislocations (GND) density map with the scale bar in $\log _{10}$ scale of dislocation lines $\mathrm{m}^{-2}$. The in-plane rotation $\omega_{12}$, and the out-ofplane rotations $\omega_{13}$ and $\omega_{23}$, rotating about the 2 and 1 axis respectively are shown.

Similar behaviour of the MC carbides was not observed in the case of OP testing. In particular, for the OP-tests, it was not found that the $\mathrm{MC}$ carbides were cracked or the MC/matrix interfaces were damaged; such observations were very rare. However, for OP-testing - with 5 and 360 minutes dwell time - cracks were found to initiate primarily at regions where transgranular $\mathrm{MC}$ carbides were oxidised close to the surface. Figures $6 \mathrm{~b}$ and $6 \mathrm{~d}$ illustrate the crack initiation sites for the OP-tests with 5 and 360 minutes dwell time, respectively. Figure 9 illustrates EDX maps collected from the crack initiation site of the OP-test with 6 hours dwell (see Figure 6d). The oxidised Ta/Ti-rich MC carbide can be observed at the crack and it is denoted by the red arrow in the Ta map. At the $\mathrm{C}$ map, the position of the oxidised carbide is denoted by the white dashed line; note that the brightest red area is due to the mounting material.

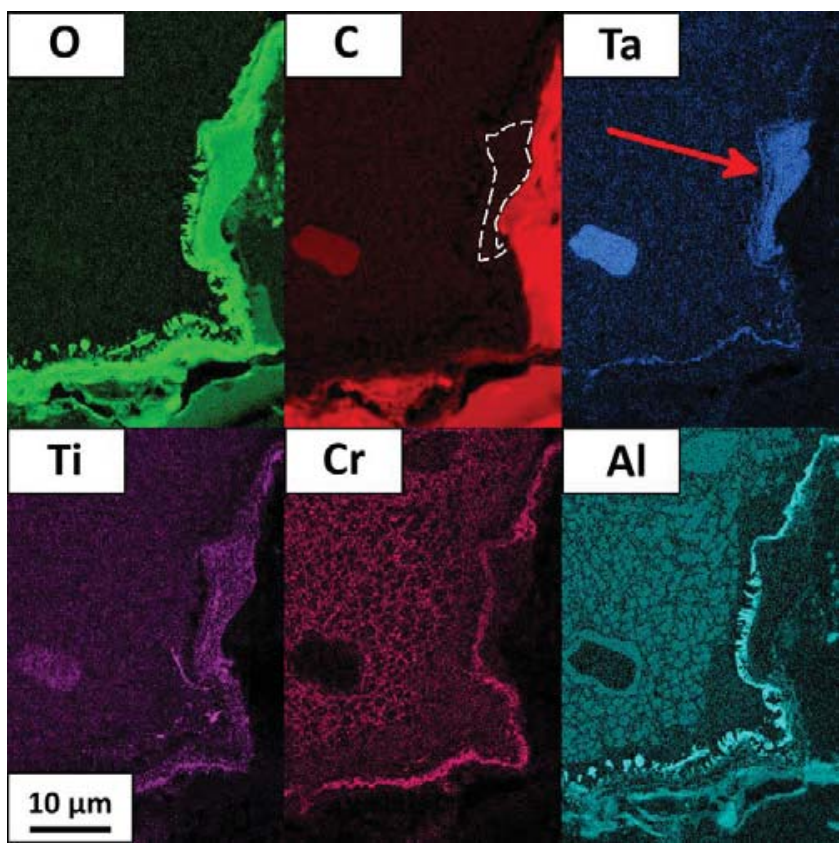

Figure 9: EDX chemical mapping of the crack initiation area as denoted by the red box in Figure $6 \mathrm{~d}$ for the OP-test with 360 minutes dwell. The crack propagates in the perpendicular direction. The red arrow indicates the oxidised $(\mathrm{Ta} / \mathrm{Ti}) \mathrm{C}$ carbide and the white dashed line shows the position of the carbide at the C map.

Based on the above microstructural observations, one would expect that the crack initiation life of IN792 would be shorter under IP conditions compared to OP. Table III summarises the cycles to crack initiation for all the investigated conditions. Crack initiation was defined as the number of cycles required to grow a crack of $100 \mu \mathrm{m}$ length. Indeed a shorter crack initiation life was obtained for specimens tested under IP conditions. Therefore, it can be suggested that the presence of MC carbides is relatively more detrimental under IP-TMF conditions, because accumulation of plasticity around the carbides leads to early fracture. But further studies are needed to confirm this conjecture.

Table III. Cycles to crack initiation for all the investigated conditions.

\begin{tabular}{|c|c|}
\hline Cycle type & Cycles to crack initiation \\
\hline IP - 5 min dwell & 77 \\
\hline IP - 360 min dwell & 82 \\
\hline OP - 5 min dwell & 95 \\
\hline OP - 360 min dwell & 102 \\
\hline
\end{tabular}



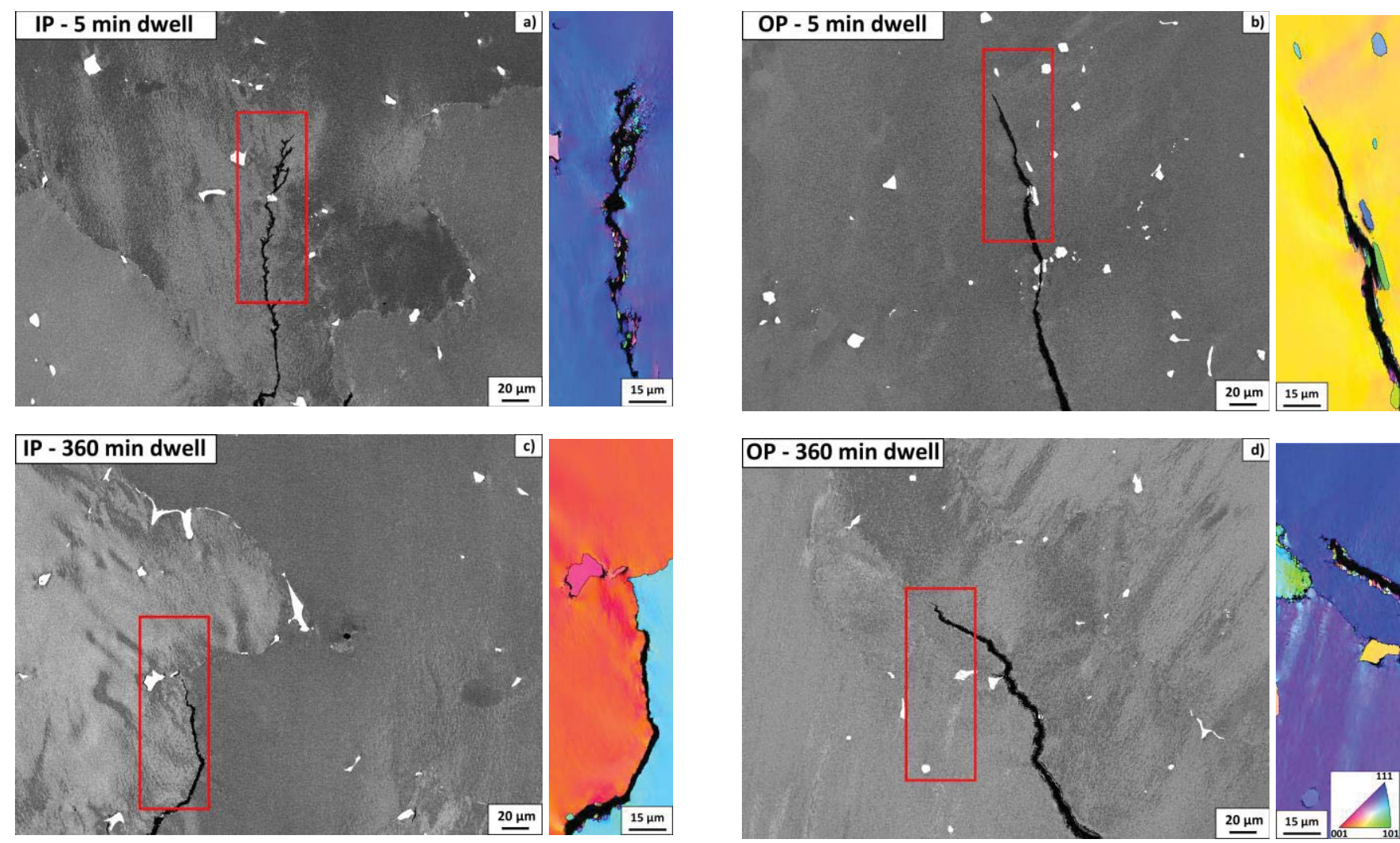

Figure 10: Backscattered SEM images showing the crack tips in a) IP-5 minutes dwell, b) OP-5 minutes dwell, c) IP-360 minutes dwell and d) OP-360 minutes dwell specimens alongside corresponding EBSD inverse pole figure maps. Red boxes denote the area scanned by EBSD. Loading axis is in horizontal direction.

\section{$\underline{\text { Crack Propagation }}$}

Microstructural observations of the cracks for each investigated condition have shown that in the case of 5 minutes dwell time cracks - in both IP and OP conditions - propagate transgranularly; representative length of the cracks are illustrated in Figure 10a and 10b. By contrast, 6 hours dwell time results in intergranular crack propagation in the case of the IP condition (Figure 10c); however crack propagation remains transgranularly in OP, as illustrated in Figure 10d and the corresponding EBSD inverse pole figure plot. Other studies on IN792 found similar behaviour for IP and OP tests between $100-750{ }^{\circ} \mathrm{C}[10]$. All of the above observations have been confirmed by EBSD scans, as shown in Figure 10.

\section{Discussion}

\section{$\underline{\text { MC Carbides - Crack Initiation Sites }}$}

The main parameters influencing the crack initiation life of IN792 have been summarised in Table II. These observations lead one to question the precise role of MC carbides and particularly carbon in polycrystalline superalloys, for high temperature applications. In addition to the post-mortem findings about the MC carbides, the EBSD strain mapping of the MC carbides in the fully heat treated microstructure raises further queries about the effect of MC carbides in these materials.
A backscattered SEM image of a grain boundary region after full heat treatment - prior to any deformation by fatigue - which contains two MC carbides are illustrated in Figure 11, alongside corresponding EBSD lattice strain maps. The red box indicates the particular area of the strain mapping analysis; two bright-contrast intergranular Ta/Ti-rich MC-type carbides can be observed.

The strain mapping analysis revealed residual thermal strains around the MC carbides, after the full heat treatment and prior to any deformation. Residual strains can be seen at the top left-hand and bottom left-hand corner of the normal strain $\varepsilon_{11}$ along the horizontal $\mathrm{x}$ axis map. Cooling from the ageing temperature combined with the differing thermal expansion coefficients between the MC carbides and the $\gamma / \gamma^{\prime}$ microstructure leads to the observed residual thermal strains. Similar behaviour has been observed previously in a directionally solidified superalloy containing $\mathrm{MC}$ carbides and in a polycrystalline nickel superalloy for disk applications containing non-metallic inclusions $[11,12]$.

From all of the above observations, we conclude that the optimal carbon content in polycrystalline superalloys must be important in determining fatigue performance [13]. For instance, the TMF crack initiation life might be potentially improved by decreasing the carbon content and subsequently lowering the volume fraction of MC carbides within the microstructure. Optimisation of the cooling process to minimise the residual thermal strains and the propensity for carbide cracking might also be important. 

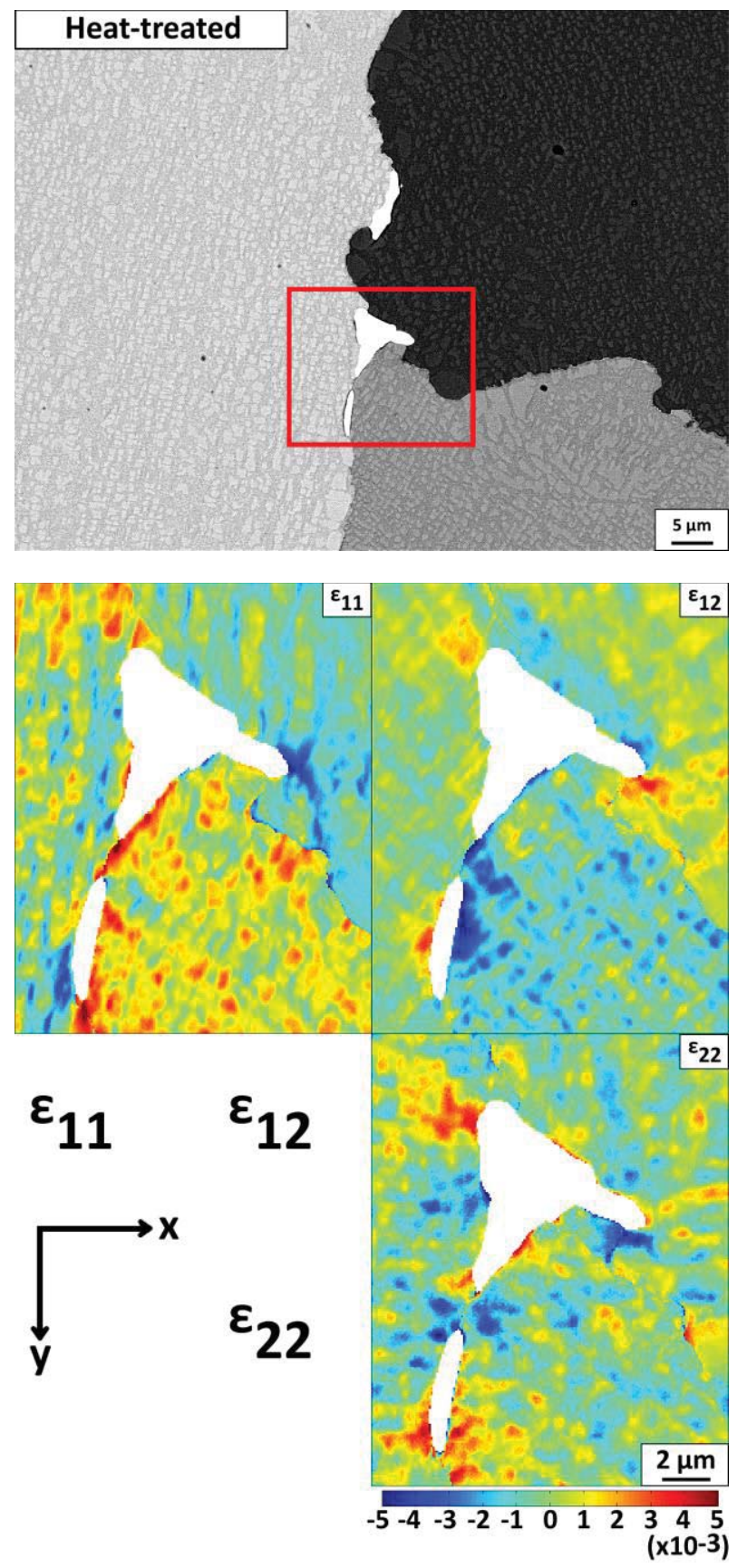

Figure 11: Backscattered SEM image of fully heat treated IN792 showing intergranular MC carbides and corresponding EBSD strain maps. The strain $\varepsilon_{11}$ along the horizontal axis $\mathrm{x}$, the in-plane shear strain $\varepsilon_{12}$ and the strain $\varepsilon_{22}$ along the vertical axis y are shown.

In the OP-tests, carbides were not observed to be cracked. However, cracks were found to initiate from oxidised MC carbides - see Figures $6 \mathrm{~b}$ and $6 \mathrm{~d}$. It is known that MC-type carbides oxidise faster than the $\gamma / \gamma^{\prime}$ microstructure [14]. Moreover, in a previous study the oxidation of MC carbides was found to result in surface eruptions in unstressed at polished specimens and that a substantial volume expansion occurs as a result of oxidation [15]. Thus, localised plastic deformation around oxidising carbides occurs and thereby they serve as crack initiation sites [16]. In the case of the OP-tests, the MC carbides oxidise during compression at the maximum temperature and subsequently crack in tension at the cold end of the cycle, when they exhibit low ductility.

Finally, one should acknowledge that the shape of the MC carbides may influence the crack initiation life. In particular, oxidising script-type MC carbides can serve as sharp oxide intrusions from which cracks can initiate. In the case of IN792, script-type MC carbides are not observed; blocky shaped MC carbides are dominant.

\section{The Effect of Dwell Time on Crack Propagation}

It was shown that the increase of dwell time dramatically increases the crack growth rate for both IP and OP conditions. Besides, a slightly higher crack growth rate was observed for the IP-test compared to the OP-test as is illustrated in Figure 4. The crack propagation character and the parameters influencing the crack propagation for each condition are summarised in Table IV and can be used to rationalise the above observations.

Table IV. Summary of crack propagation mode and parameters controlling the crack propagation for all the investigated conditions.

\begin{tabular}{|c|c|c|}
\hline & IP-TMF & OP-TMF \\
\hline 5 min dwell & $\begin{array}{c}\text { Transgranular } \\
\text { propagation - } \\
\text { Oxidation damage }\end{array}$ & $\begin{array}{c}\text { Transgranular } \\
\text { propagation - } \\
\text { Oxidation damage }\end{array}$ \\
\hline 360 min dwell & $\begin{array}{c}\text { Intergranular } \\
\text { propagation - } \\
\text { Grain boundary } \\
\text { character }\end{array}$ & $\begin{array}{c}\text { Transgranular } \\
\text { propagation - } \\
\text { Oxidation assisted } \\
\text { recrystallisation }\end{array}$ \\
\hline
\end{tabular}

IP-TMF and Grain Boundary Character: In the case of the IP-tests, the increase of dwell time led to a change in crack propagation mode; a transition from transgranular to intergranular crack propagation was observed. Thus, the higher crack growth rate is attributed to the different crack propagation character.

The transition from transgranular to intergranular crack propagation for the IP-tests can be rationalised as follows. For short dwell times (5 minutes), diffusion phenomena such as oxidation and cavity formation at grain boundaries are not sufficient to impact the crack growth rate. However, the introduction of longer dwell times - i.e. 360 minutes - results in stress relaxation, oxidation and creep cavitation - consequently enhanced creep damage, particularly at grain boundaries. Thereby, creep damage forming at grain boundaries can interact with the main crack and lead to higher crack growth rates. It is apparent that the grain boundary character of the alloy will determine how catastrophic the increase of dwell time will be to the TMF performance. 
One of the main factors influencing the grain boundary character of superalloys is the precipitation of phases such as different types of carbides. As it was shown, cavities were found to form at the MC carbides with $\gamma / \gamma^{\prime}$ interfaces at the grain boundaries. Voids or cracked MC carbides were observed even ahead of the crack tip as illustrated in Figure 7. Thereby the grain boundaries become more susceptible to failure and thus the crack growth rate can be increased.
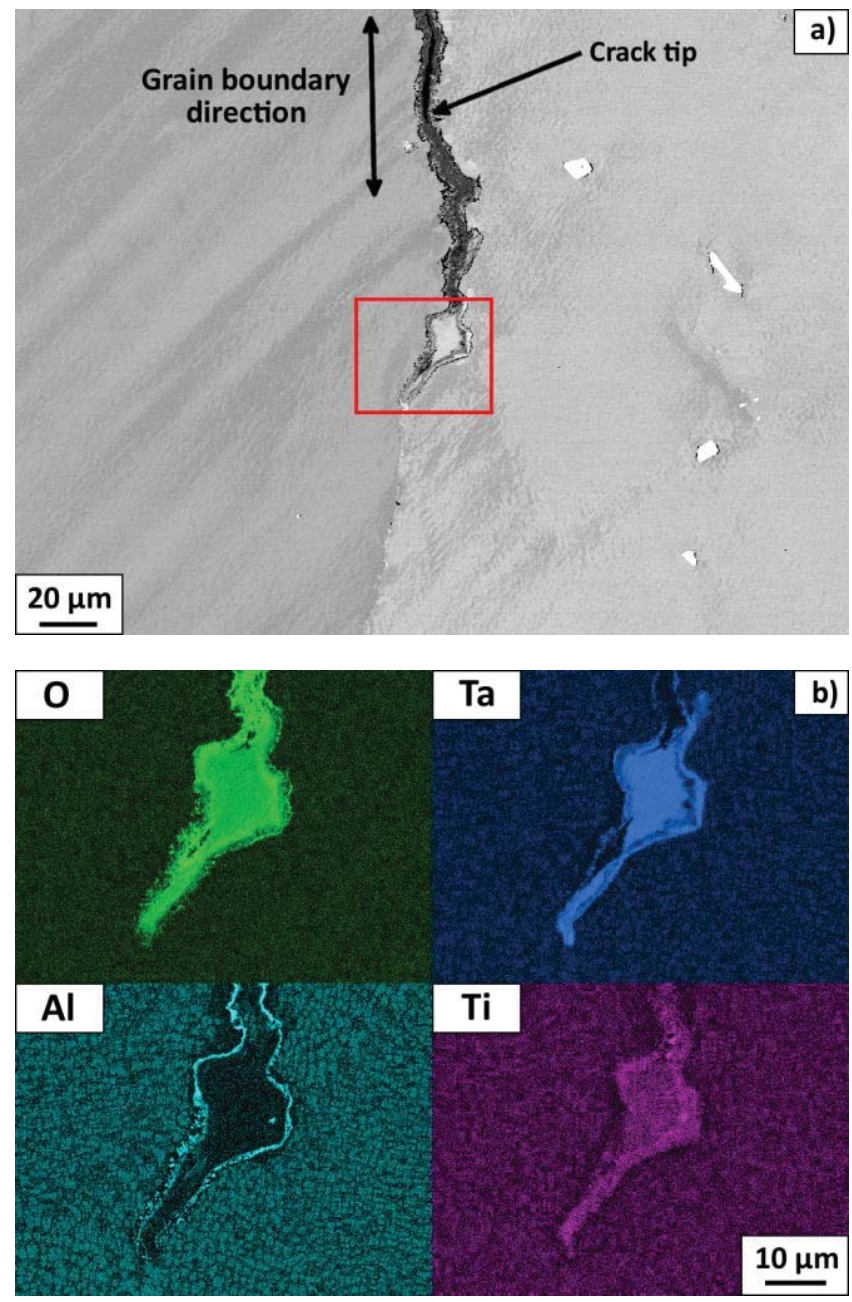

Figure 12: a) Backscattered SEM image of the secondary crack tip of the IP-360 minutes dwell time specimen and b) EDX analysis of the intergranular oxidised MC carbide. The red box denotes the area analysed by EDX.

Besides, it was mentioned earlier that MC carbides oxidise faster than the $\gamma / \gamma^{\prime}$ microstructure. An atom probe study on a polycrystalline superalloy for industrial gas turbines has shown $\mathrm{Cr}$ and $\mathrm{Al}$ segregation at the grain boundary interfaces $\mathrm{MC} / \gamma$ and $\mathrm{MC} / \gamma^{\prime}$ [17]. Segregation of these elements, combined with higher diffusion rates along the grain boundaries, compared to those in the grain interior can result in higher oxidation rates at these particular interfaces; relatively fast crack propagation rates are facilitated. In addition, neither boron nor carbon were found to segregate at these interfaces. Their presence, particularly of boron, could potentially decrease grain boundary diffusivity and oxidation damage.

Figure 12a illustrates a backscattered image of the secondary intergranular crack tip where the intergranular oxidised MC carbide can be seen ahead of the crack tip; EDX elemental maps of the carbide are illustrated in Figure 12b. The chemistry of the MC carbides and subsequently their thermal stability can influence crack propagation; less stable MC carbides will decompose and oxidise faster, leading to grain boundaries more prone to failure.

OP-TMF and Oxidation Performance: In the case of the OP-tests, although a transition from transgranular to intergranular crack propagation is not observed, a higher crack growth rate occurs as the dwell time increases to 6 hours. The increase of the crack growth rate in the OP-test is the result of the stress response of the material to the increased dwell time.

In particular, as the holding time under compression at the maximum temperature increases, stress relaxation occurs. As a consequence, greater tensile mean stress is developed. It is apparent that as the holding time increases, the higher the tensile mean stress at the cold end of the TMF cycle. Hence, the higher crack growth rate observed in the OP-test with a 6 hours dwell is ascribed to the higher developed tensile mean stress for the given investigated strain range. Figure 5 illustrates the minimum and maximum forces, $N$, as a function of crack length, respectively. It can be seen that the maximum force increases as dwell increases in the OP tests; similar behaviour is not observed for the IP tests.

However, a microstructural factor that can potentially influence the crack growth rate under OP conditions as dwell time increases is related to the oxidation performance of the alloy. At the OP-test with a 6 hours dwell time, extensive recrystallisation enhanced by oxidation is observed along the crack. A $\gamma^{\prime}$ denuded zone is present due to oxidation, where recrystallisation can occur without the retarding effect of $\gamma^{\prime}$ precipitates. Although recrystallisation at the $\gamma^{\prime}$ denuded zones is observed for all conditions, it is observed relatively far behind the crack tip. In the case of the OP-test with a 6 hours dwell, recrystallisation occurs in the vicinity of the crack tip as illustrated in Figure 13; confirmed by EBSD analysis. It is apparent that the oxidation resistance of the alloy and the formation rate of the $\gamma^{\prime}$ denuded zone can control the crack growth rate at the OP-TMF case with a dwell period. Besides, as the maximum temperature increases, the effect of dwell on TMF performance, both IP and OP, will be enhanced and crack propagation rates will increase.

In consideration of all of the observed facts, it is concluded that the increase of dwell time exerts a stronger effect under IP-TMF conditions than in OP conditions. 

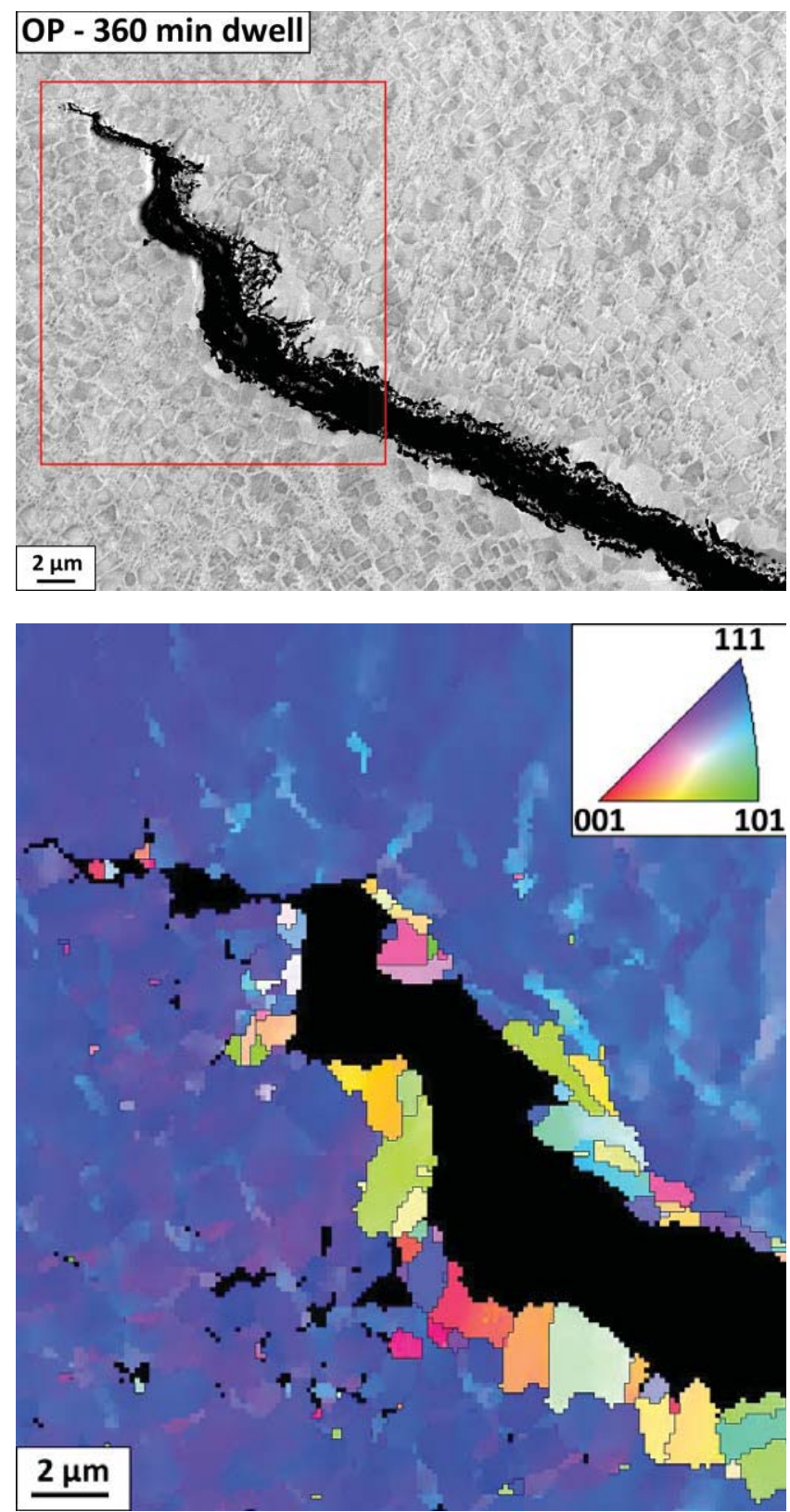

Figure 13: Crack tip from the OP with 360 minutes dwell time specimen, alongside corresponding inverse pole map (as denoted by the red box) showing the recrystallisation enhanced by oxidation.

\section{Conclusions}

In-phase (IP) and out-of-phase (OP) TMF tests were performed on the conventionally cast superalloy IN792, with 5 minutes and 6 hours dwell time at the maximum investigated temperature $\left(750{ }^{\circ} \mathrm{C}\right)$. The main conclusions can be summarised as follows:

- $\quad$ For a 5 minute dwell, the crack growth rates for IP and OP testing were broadly similar. But 6 hours dwell time increases dramatically the crack growth rate; IP testing gives rise to slightly higher crack growth rates than OP.
- Under IP conditions, a transition from transgranular to intergranular crack propagation was observed as the dwell time increases. The transgranular mode of crack propagation under OP conditions was not altered by an increase in dwell time.

- Residual thermal strains were observed by EBSD strain mapping around $\mathrm{MC}$ carbides in fully heat treated microstructure prior to deformation. It was shown that MC carbides can serve as crack initiation sites under IP and OP conditions.

- A substantial fraction of the MC carbides were found to have cracked after IP testing; voids were also observed to form at the interface between MC and the $\gamma / \gamma^{\prime}$ microstructure. The significant accumulation of plasticity around cracked MC carbides was confirmed by EBSD strain mapping.

- In the OP-tests, oxidised MC carbides close to the surface were found to serve as crack initiation sites.

\section{Acknowledgements}

The work has been financially supported by Siemens Industrial Turbomachinery $\mathrm{AB}$ in Finspång, Sweden and the Swedish Energy Agency, via the Research Consortium of Materials Technology for Thermal Energy Processes, Grant No. KME-702. The authors also thank Gabriella Chapman for her assistance with the EDX mapping.

\section{References}

1. J.J. Moverare, S. Johansson, and R.C. Reed, "Deformation and damage mechanisms during thermal-mechanical fatigue of a single-crystal superalloy," Acta Materialia, 57 (2009), 2266-2276.

2. J.J. Moverare and D. Gustafsson, "Hold-time effect on the thermo-mechanical fatigue crack growth behaviour of Inconel 718," Materials Science and Engineering: A, 528 (2011), 86608670 .

3. J.J. Moverare et al., "Thermomechanical fatigue crack growth in a cast polycrystalline superalloy," MATEC Web of Conferences, 14 (2014), 19004.

4. A.J. Wilkinson, G. Meaden, and D.J. Dingley, "High-resolution elastic strain measurement from electron backscatter diffraction patterns: New levels of sensitivity," Ultramicroscopy, 106 (45) (2006), 307-313.

5. S. Guth et al., "Influence of dwell times on the thermomechanical fatigue behavior of a directionally solidified Ni-base superalloy," International Journal of Fatigue, 80 (2015), 426-433. 
6. Z.W. Huang et al., "Thermomechanical fatigue behavior and life prediction of a cast nickel-based superalloy," Materials Science and Engineering: A, 432 (2006), 308-316.

7. F. Liu et al., "Thermal-mechanical fatigue behavior of a cast K417 nickel-based superalloy," International Journal of Fatigue, 24 (8) (2002), 841-846.

8. M.J. Starink and P.A.S. Reed, "Thermal activation of fatigue crack growth: Analysing the mechanisms of fatigue crack propagation in superalloys," Materials Science and Engineering: A, 491 (1-2) (2008), 279-289.

9. M.M. Kirka et al., "Parameters influencing thermomechanical fatigue of a directionally-solidified Ni-base superalloy," International Journal of Fatigue, 81 (2015), 48-60.

10. J. Kanesund, J.J. Moverare, and S. Johansson, "Deformation and damage mechanisms in IN792 during thermomechanical fatigue," Materials Science and Engineering: A, 528 (2011), 4658-4668.

11. P.S. Karamched and A.J. Wilkinson, "High resolution electron back-scatter diffraction analysis of thermally and mechanically induced strains near carbide inclusions in a superalloy," Acta Materialia, 59 (1) (2011), 263-272.

12. T. Zhang et al., "Crystal plasticity and high-resolution electron backscatter diffraction analysis of full-field polycrystal $\mathrm{Ni}$ superalloy strains and rotations under thermal loading," Acta Materialia, 80 (2014), 25-38.

13. J. Yang et al., "Effects of different $\mathrm{C}$ contents on the microstructure, tensile properties and stress-rupture properties of IN792 alloy," Materials Science and Engineering: A, 528 (3) (2011), 1534-1539.

14. J. Litz, A. Rahmel, and M. Schorr, "Selective carbide oxidation and internal nitridation of the Ni-base superalloys IN738LC and IN939 in air," Oxidation of Metals, 30 (1-2) (1988), 95-105.

15. T. Connolley, P.A.S Reed, and M.J. Starink, "Short crack initiation and growth at $600{ }^{\circ} \mathrm{C}$ in notched specimens of Inconel 718," Materials Science and Engineering: A, 340 (2003), 139154.

16. A. Pineau et al., "Failure of metals II: Fatigue," Acta Materialia, 107 (2016), 484-507.

17. P. Kontis et al., "On the effect of boron on grain boundary character in a new polycrystalline superalloy," Acta Materialia, 103 (2016), 688-699. 